Sind die Probleme der

Bevölkerungsalterung durch eine höhere

Geburtenrate lösbar?

Barbara Berkel, Axel Börsch-Supan,

Alexander Ludwig und Joachim Winter

25-2002

September 2002 


\title{
Sind die Probleme der Bevölkerungsalterung durch eine höhere Geburtenrate lösbar?
}

\author{
Barbara Berkel*, Axel Börsch-Supan****, Alexander Ludwig*, Joachim Winter* \\ * Mannheim Research Institute for the Economics of Aging (MEA), Universität Mannheim \\ ** National Bureau for Economic Research (NBER), Cambridge, Mass.
}

September 2002

\section{Zusammenfassung}

Hilft eine höhere Geburtenrate, die Folgen der Alterung zu dämpfen? Die Ergebnisse der Wirtschaftstheorie sind keinesfalls eindeutig, auch wenn die Idee - „Wenn wir zu viele Alte haben, brauchen wir mehr Kinder, um dies wieder auszugleichen“" - plausibel erscheint. Auch die vorliegende quantitative Studie, die auf einem makroökonomischen Simulationsmodell für Deutschland basiert, kommt zu einem differenzierten Ergebnis: Eine langfristige Stärkung des Bruttonationaleinkommens pro Kopf entsteht durch eine höhere Geburtenrate nur dann, wenn die zusätzlich geborenen Kinder auch besser ausgebildet werden. Entscheidend für ein langfristiges Wachstum ist daher das zukünftige Humankapital. Darüber hinaus ist die Dauer der Übergangszeit, nach deren Ablauf sich eine höhere Geburtenrate in mehr und besser ausgebildeten Erwerbstätigen niederschlägt, die in die Renten- und anderen Sozialversicherungskassen einzahlen können, sehr lang. Von daher haben weitere Reformen unserer Sozialversicherungen nach wie vor höchste Priorität, da nur sie die sozialpolitischen Probleme einer alternden Babyboomgeneration kurz- und mittelfristig lösen können.

Adresse: MEA, Universität Mannheim

D-68131 Mannheim

Fax: +49-0621-181-1861

www.mea.uni-mannheim.de

Wir danken dem Heidelberger Büro für Familienfragen und soziale Sicherheit sowie dem Gesamtverband der deutschen Versicherungswirtschaft, dem Land Baden-Württemberg und der Volkswagen-Stiftung für ihre finanzielle Unterstützung, Hendrik Engelmann-Pilgers für die hervorragende Assistenz und Florian Heiss für hilfreiche Anmerkungen. 


\section{Einleitung}

Der sich allmählich beschleunigende demographische Wandel gehört zu den wichtigsten gesellschaftlichen Entwicklungen der Zukunft. Durch den Alterungsprozeß nimmt die Anzahl der Personen im Ruhestand zu, während die erwerbstätige Bevölkerung zurückgeht. Eine steigende Alterslast wird somit in der Zukunft auf immer weniger Schultern verteilt. Dies führt zu einem wachsenden Druck auf die Systeme der sozialen Sicherung und auf die Gesamtwirtschaft.

Bislang werden vor allem die Konsequenzen der Alterung diskutiert, insbesondere die Finanzierung und Ausgestaltung der gesetzlichen Rentenversicherung. Es liegt jedoch nahe, auch die Ursachen der Bevölkerungsalterung anzugehen. Dabei rückt die immer weiter sinkende Geburtenrate in den Vordergrund. Die Frage liegt auf der Hand: Ist das Problem der Alterung durch eine höhere Geburtenrate lösbar?

Die Wirtschaftstheorie gibt auf diese Frage keine eindeutige Antwort. Gemäß der neoklassischen Wachstumstheorie besteht langfristig ein negativer Zusammenhang zwischen der Wachstumsrate einer homogenen Bevölkerung und der Pro-Kopf-Produktion, da die ProKopf-Produktion eines zusätzlichen Arbeitnehmers mit der Anzahl der Arbeitnehmer eines Landes sinkt (Solow 1956). Diese komparativ-statische Sichtweise wird jedoch den komplexen Zusammenhängen zwischen der Bevölkerungsstruktur und der gesamtwirtschaftlichen Entwicklung nicht gerecht. Kurz- und mittelfristig besteht - insbesondere während des demographischen Wandels - zwischen der Geburtenrate und dem Wirtschaftswachstum kein eindeutiger Zusammenhang. Unter Ökonomen und Demographen wird seit langem und ohne eindeutiges Ergebnis debattiert, ob eine Änderung des Bevölkerungswachstums das Wirtschaftswachstum behindert, fördert oder überhaupt nicht beeinflusst. ${ }^{1}$

Die wesentlichen Faktoren, die bei der Klärung dieser Fragestellung aus wirtschaftswissenschaftlicher Sicht berücksichtigt werden müssen, sind schnell skizziert. Eine höhere Geburtenrate kann die durch den Alterungsprozess bedingte Belastung durch Steuern und Sozialabgaben reduzieren. Darüber hinaus ist es möglich, dass eine höhere Geburtenrate das Humankapital einer Gesellschaft erhöht und somit ein positiver Wachstumseffekt entsteht (Lucas 1988, Romer 1986). Andererseits verursachen Kinder kurzfristig Kosten (vor allem für deren Ausbildung), die von der Gesellschaft zu tragen sind (Cutler et al. 1990, Weil 1999). Im Detail sind diese Mechanismen allerdings hoch komplex.

Insgesamt sind in der Theorie die Auswirkungen einer höheren Geburtenrate auf das Wirtschaftswachstum im Rahmen einer alternden Gesellschaft wegen dieser gegenläufigen Effekte unklar, so dass eine quantitative Analyse erforderlich ist. Es liegt nahe, die Auswirkungen einer höheren Geburtenrate auf das Wirtschaftswachstum mittels einer empirischen Ex-PostAnalyse zu ermitteln. Eine solche Vorgehensweise würde allerdings aus mehreren Gründen zu keinen zufriedenstellenden Ergebnissen führen:

(1) Der Alterungsprozeß ist historisch einmalig. Die alternde Gesellschaft ist ein Phänomen des späten 20. Jahrhunderts. Bislang hat noch keine Volkswirtschaft den Alterungsprozess vollständig durchlaufen.

(2) Der beobachtbare Zeitraum des derzeitigen Alterungsprozesses reicht nicht aus, um die Auswirkungen einer Veränderung der Geburtenrate auf das Wirtschaftswachstum für Deutschland in einer vergangenheitsbezogenen Untersuchung darzustellen. Der Alterungsprozess resultiert aus den niedrigen Geburtenraten seit Anfang der 70er Jahre und

\footnotetext{
${ }^{1}$ Eine Übersicht findet sich bei Bloom et al. (2001).
} 
ist daher erst eine Generation alt. Zudem vergehen 20 bis 25 Jahre, bis eine Veränderung der Geburtenrate Auswirkungen auf die gesamtwirtschaftliche Produktion hat, denn Kinder müssen zunächst aufgezogen und ausgebildet werden, bevor sie durch ihre Erwerbstätigkeit zum Bruttonationaleinkommen (BNE) ${ }^{2}$ beitragen können.

(3) Die Interaktionen zwischen Geburtenrate und Wirtschaftswachstum sind sehr komplex und dürften daher aus einer sich auf eine relativ kurze Vergangenheit stützende Untersuchung kaum erschließbar sein.

Aus diesen Gründen ist ein makroökonomisches Simulationsmodell für die vorliegende Fragestellung besser geeignet. Es kann die komplexen Zusammenhänge zwischen dem Alterungsprozess und makroökonomischen Variablen wie dem Pro-Kopf-Wirtschaftswachstum über einen Zeitraum von mehr als einer Generation strukturell abbilden. In einem solchen Modell können verschiedene Geburtenraten simuliert und ihre Auswirkungen auf das Wirtschaftswachstum über den Zeitraum 2000 bis 2100 berechnet werden. Dieser lange Zeitraum ist nötig, denn neben kurzfristigen müssen auch sehr langfristige Entwicklungen betrachtet werden. Um die Auswirkungen einer Veränderung der Geburtenrate vollständig erfassen zu können, muss man sie mindestens über die Dauer eines Menschenlebens verfolgen. ${ }^{3}$

Tabelle 1: Annahmen zur Geburtenrate

\begin{tabular}{cccccccc}
\hline & \multicolumn{2}{c}{ fallend } & \multicolumn{2}{c}{ konstant } & \multicolumn{2}{c}{ steigend } \\
& West & Ost & West & Ost & West & Ost \\
\hline $\mathbf{1 9 9 9}$ & 1,4 & 1,14 & 1,4 & 1,14 & 1,4 & 1,14 \\
$\mathbf{2 0 1 5}$ & 1,1 & 1,1 & 1,4 & 1,4 & 1,8 & 1,8 \\
$\mathbf{2 1 0 0}$ & 1,1 & 1,1 & 1,4 & 1,4 & 1,8 & 1,8 \\
\hline
\end{tabular}

Quelle: Statistisches Bundesamt (2001) für 1999; die Werte für 2015 und 2100 beruhen auf unseren Fertilitätsszenarien.

Das in der vorliegenden Untersuchung verwendete Simulationsmodell ${ }^{4}$ besteht aus drei Komponenten, die aufeinander aufbauen: einer Bevölkerungsprognose, einer Erwerbstätigenprognose und einem auf diesen beiden Prognosen aufbauenden makroökonomischen Modell. Zur Analyse der gesamtwirtschaftlichen Effekte einer Veränderung der Geburtenrate bilden wir in der Bevölkerungsprognose drei Fertilitätsszenarien ab. Im Ausgangsszenario gehen wir davon aus, dass die gesamtdeutsche Geburtenrate konstant bei derzeit etwa 1,36 Kindern pro Frau bleibt. Dieses Ausgangsszenario kontrastieren wir mit einem Anstieg der Geburtenrate auf 1,8 Kinder pro Frau. Eine Geburtenrate dieser Größenordnung kann man derzeit in Frankreich $(1,8)$, in einigen skandinavischen Ländern (Dänemark 1,65, Norwegen 1,70) und in den Vereinigten Staaten $(1,93)$ finden. Daneben untersuchen wir auch ein mögliches weiteres Absinken der Geburtenrate auf 1,1 Kinder pro Frau. Dies entspricht ungefähr der Geburtenrate einiger südeuropäischer Länder (Spanien 1,13 oder Italien 1,20) sowie vieler osteuropäischer Länder (z.B. Bulgarien 1,10, Tschechische Republik 1,16). Die Erwerbstätigenprognose ba-

\footnotetext{
${ }^{2}$ Früher Bruttosozialprodukt (BSP).

${ }^{3}$ Guest und McDonald (2002) untersuchen eine ähnliche Fragestellung ebenfalls anhand eines Simulationsmodells. Sie betrachten die Auswirkungen einer fallenden Geburtenrate auf den australischen Lebensstandard.

${ }^{4}$ Eine umfassende und detaillierte Darstellung der Methodik findet sich im Gutachten für das Heidelberger Büro für Familienfragen und soziale Sicherheit (Börsch-Supan, Berkel, Ludwig und Winter, 2002).
} 
siert auf der Bevölkerungsprognose und Annahmen bezüglich der zukünftigen alters- und geschlechtsspezifischen Erwerbsbeteiligung. Die Bevölkerungs- und Erwerbstätigenprognose, dargestellt in Abschnitt 2, liefern die exogenen Daten für das makroökonomische Simulationsmodell.

Die Beschreibung des makroökonomischen Simulationsmodells - ein Mehr-Länder-Modell mit überlappenden Generationen - folgt in Abschnitt 3. Daraus ergeben sich die für unsere Fragestellung relevanten makroökonomischen Größen wie das BNE Deutschlands und dessen Wachstumsrate. Diese Ergebnisse werden schließlich in Abschnitt 4 diskutiert.

\section{Die zukünftige Entwicklung der Bevölkerung und der Erwerbstätigen}

Ausgangspunkt unserer Simulationen sind die sich bezüglich der Geburtenrate unterscheidenden Varianten der Bevölkerungsprognose und die sich daraus ergebenden Erwerbstätigkeitsprognosen. Das Bevölkerungsmodell für Deutschland ergibt sich aus einem nach Lebensjahren und Geschlecht differenzierten Fortschreibungsverfahren. In dem Modell können verschiedene Szenarien der Geburtenrate, Mortalität und Migration miteinander kombiniert werden, wobei wir in diesem Beitrag auf eine Variation der Geburtenrate beschränken.

Im Vergleichsszenario wird die in Westdeutschland seit über zwei Jahrzehnten nahezu konstante Geburtenrate von 1,4 Kindern pro Frau unverändert fortgeschrieben. Zudem wird angenommen, dass die Geburtenrate der ostdeutschen Frauen sich von derzeit 1,15 bis 2015 kontinuierlich an den westdeutschen Wert anpasst. Im Vergleich zu dem Szenario einer konstanten Geburtenrate betrachten wir die in der Einleitung kurz angerissenen alternativen Szenarien, die Geburtenraten in anderen europäischen Ländern reflektieren. Zum einen gehen wir davon aus, dass die Geburtenrate bis 2015 von 1,4 auf 1,8 Kinder je Frau ansteigt. Zum anderen unterstellen wir ein ungefähr symmetrisches Absinken der Geburtenrate auf 1,1 Kinder je Frau. Diese zwei Szenarien dienen dazu, die Bandbreite der Effekte einer Variation in der Geburtenrate auf das Wirtschaftswachstum zu verdeutlichen. ${ }^{5}$

Hinsichtlich der Annahmen zur Lebenserwartung und Erwerbstätigenentwicklung für Deutschland orientieren wir uns an den mittleren Prognosen von Birg und Börsch-Supan (1999). Diese Größen sind in den alten und neuen Bundesländern zunächst noch unterschiedlich, sie gleichen sich jedoch im Zeitablauf an. Lediglich bei der Migration weichen wir von Birg und Börsch-Supan (1999) ab und schreiben das Verhältnis der in 1999 Immigrierten zur deutschen Gesamtbevölkerung für die Zukunft fort. Veränderungen in der Bevölkerung können somit eindeutig auf Unterschiede in den Fertilitätsszenarien zurückgeführt werden.

Da wir in unserem makroökonomischen Simulationsmodell auch internationalen Kapitalverkehr zulassen und damit auch andere Länder modellieren, benötigen wir auch Bevölkerungsund Erwerbstätigkeitsprognosen für das Ausland (übrige EU-Länder). Dazu verwenden wir Projektionen der Vereinten Nationen sowie die Labor Force Statistics der OECD (UN 2000, OECD 1999).

Unter Annahme einer konstanten Geburtenrate von 1,4 Kindern je Frau würde Deutschlands Bevölkerung innerhalb von 100 Jahren von heute ca. 82 Mio. Einwohnern auf knapp die Hälfte schrumpfen (Abbildung 1). Eine um 30 Prozent höhere Geburtenrate von 1,8 Kindern je Frau würde der Bevölkerungsrückgang deutlich dämpfen, aber nicht ausreichen, um die Be-

\footnotetext{
${ }^{5}$ Neben diesen Szenarien mit bis 2015 sehr schnell steigenden bzw. fallenden Geburtenraten haben wir auch bis 2030 langsamer ansteigende bzw. fallende Geburtenraten untersucht. Allerdings sind im Ergebnis die Unterschiede dieser beiden Alternativen zu den hier vorgestellten Varianten mittelfristig vernachlässigbar; vgl. Börsch-Supan, Berkel, Ludwig und Winter (2002).
} 
völkerung zu stabilisieren, da dieser Wert unter dem Bestandserhaltungsniveau von 2,1 Kindern je Frau liegt. Analog zur Entwicklung der Gesamtbevölkerung verhält sich die Entwicklung der Anzahl der Kinder, die hier als alle unter 20jährigen definiert sind (Abbildung 1).

\section{Abbildung 1: Bevölkerungsentwicklung und Anzahl der Kinder}
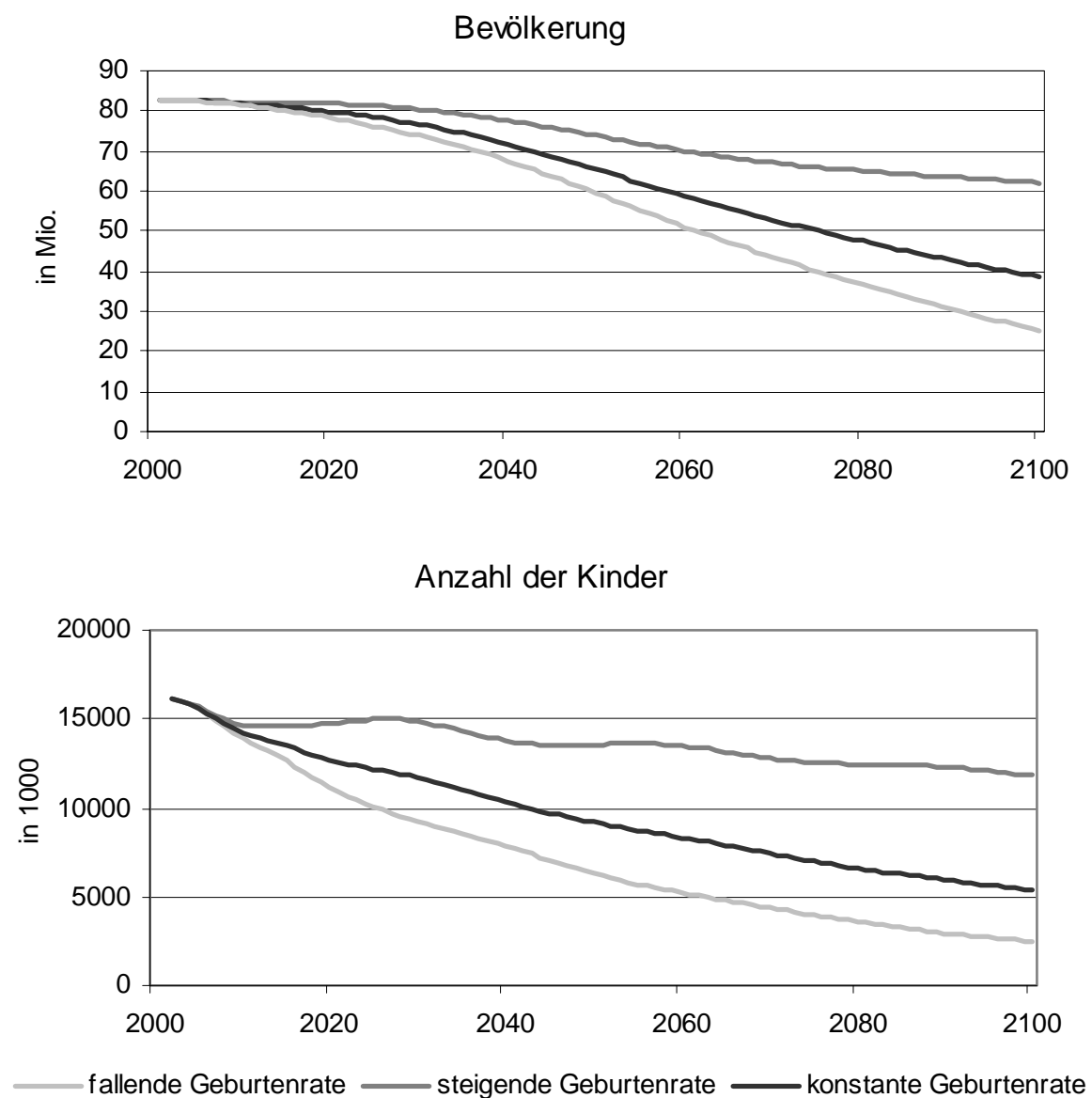

In den ersten 10 Jahren unterscheidet sich die Kinderzahl der einzelnen Szenarien kaum, da die Geburtenrate zunächst das neue Niveauerreichen muss. Langfristig schrumpfen in allen Szenarien sowohl die Gesamtbevölkerung als auch die Anzahl der Kinder - bei fallender Geburtenrate deutlich schneller als bei einer steigenden Geburtenrate.

Die bei einer höheren Geburtenrate zusätzlich geborenen Kinder werden (im Mittel) nach 20 Jahren erwerbstätig und vergrößern erst dann das Arbeitsangebot. Daher wirkt sich eine Veränderung der Geburtenrate von 2002 bis 2015 erst ab 2035 auf die Anzahl der Erwerbstätigen aus (Abbildung 2).

Sehr deutlich erkennbar ist das Problem des alternden Deutschlands: Die Anzahl der Rentner steigt bis 2035 nicht nur absolut, sondern auch relativ weiter an, wie aus dem Rentnerquotienten (Abbildung 3) deutlich wird. ${ }^{6}$ Dieser dramatische Anstieg entsteht, weil die in den 60er Jahren geboren Babyboomer ab 2020 das Rentenalter erreichen. Unter der Annahme einer steigenden Geburtenrate fällt der Rentnerquotient ab 2035 wieder, bei konstanter Gebur-

\footnotetext{
${ }^{6}$ Der Rentnerquotient ist definiert als Anzahl der Rentner relativ zur Anzahl der Erwerbstätigen.
} 
tenratenrate stagniert er. Sollte die Geburtenrate gar weiter zurückgehen, so steigt der Rentnerquotient auch nach 2040 weiter an.

\section{Abbildung 2: Anzahl der Erwerbstätigen und Rentner}
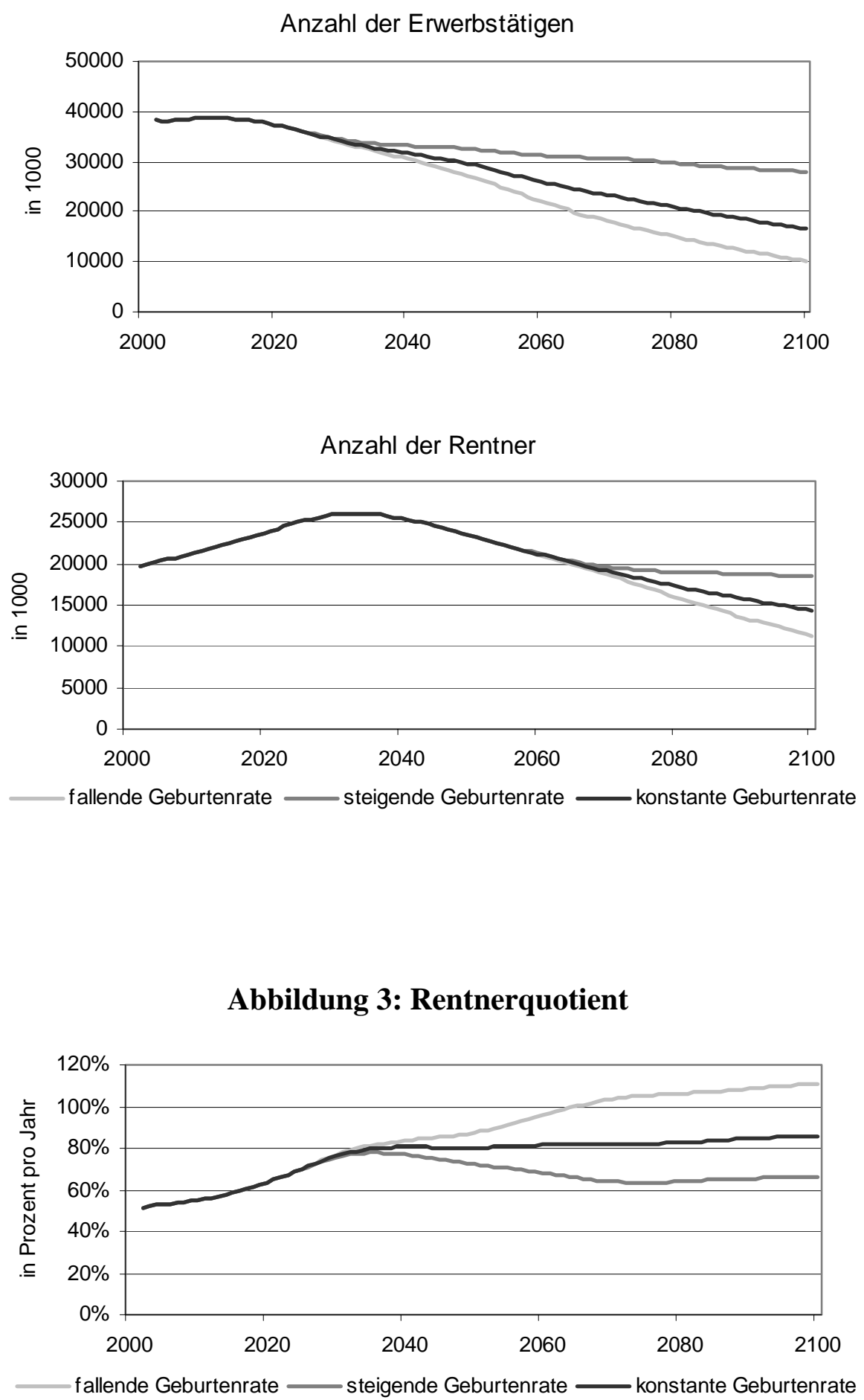

Die Abbildungen 1 bis 3 haben die folgende wichtige Botschaft: Selbst bei einer rasch ansteigenden Geburtenrate ist das Alterungsproblem bis 2035 unausweichlich. Kurzfristig gäbe es in diesem Fall insgesamt sogar einen noch größeren Anteil abhängiger Personen, da diese 
Kinder von der Erwerbsbevölkerung versorgt werden müssen. Daher kann man langfristig erst nach einem vorläufigen Gipfeln der Alterung um 2035 positive Effekte auf die Gesamtwirtschaft erwarten.

Die Abbildungen 1 bis 3 haben die folgende wichtige Botschaft: Selbst bei einer rasch ansteigenden Geburtenrate ist das Alterungsproblem bis 2035 unausweichlich. Kurzfristig gäbe es in diesem Fall insgesamt sogar einen noch größeren Anteil abhängiger Personen, da diese Kinder von der Erwerbsbevölkerung versorgt werden müssen. Daher kann man langfristig erst nach einem vorläufigen Gipfeln der Alterung um 2035 positive Effekte auf die Gesamtwirtschaft erwarten.

\section{Wie kann der Einfluss der Bevölkerungsentwicklung auf die deutsche Volkswirt- schaft modellhaft abgebildet werden?}

Vor dem Hintergrund unserer Fragestellung sind die gesamtwirtschaftlichen Auswirkungen des demographischen Wandel von besonderer Bedeutung. Wir betten daher die demographischen Szenarien des Abschnitts 2 in ein Modell der deutschen Volkswirtschaft ein. Die Erwerbstätigenprognose aus dem vorherigen Abschnitt gibt dabei das Arbeitsangebot in der Zukunft vor. Diese Größe ist in unserem Modell eine zentrale Determinante des uns interessierenden BNE pro Kopf und dessen Wachstums.

Wir betrachten ein Modell sich überlappender Generationen (OLG-Modell), das sich besonders zur Untersuchung demographischer Veränderungen eignet. OLG-Modelle gehen in ihren Ursprüngen auf Samuelson (1958) sowie Diamond (1965) zurück. Die im folgenden vorgestellte Variante ist eine Erweiterung des Modells von Auerbach und Kotlikoff (1987, Kapitel 3) zu einem Mehrländermodell, wodurch der Verflechtung nationaler Volkswirtschaften durch einen internationalen Kapitalmarkt und -güter Rechnung getragen wird (vgl. BörschSupan, Ludwig, Winter, 2002; Ludwig 2002). ${ }^{7}$

Dabei nehmen wir an, dass zwischen den betrachteten Ländern vollkommene Kapitalmärkte existieren und Kapital frei über Staatsgrenzen hinweg fließen kann. Da im folgenden die wirtschaftliche Entwicklung Deutschlands unter Berücksichtigung der Verflechtungen mit den anderen EU-Staaten betrachtet wird, ist die Annahme vollkommener Kapitalmobilität eine gute Approximation der Realität, insbesondere, nachdem die Einführung des Euro zu einer endgültigen Abschaffung der Wechselkursrisiken innerhalb der Eurozone geführt hat. Je mehr Regionen jedoch berücksichtigt werden, desto weiter entfernt sich die Annahme vollkommener Kapitalmärkte von der Realität. ${ }^{8}$

\subsection{Die Struktur unseres Modells}

In unserem Modell hat die Volkswirtschaft drei Sektoren: den Haushaltssektor, den Produktionssektor und den (rudimentären) Staatssektor, an dem uns vor allem die umlagefinanzierte Rentenversicherung interessiert.

\footnotetext{
${ }^{7}$ Modelle überlappender Generationen sind weit verbreitet, um die gesamtwirtschaftlichen Auswirkungen der Bevölkerungsalterung zu untersuchen, siehe Kotlikoff, Smetters und Walliser (1999, 2001), De Nardi et al. (1999) und Altig et al. (2001) für die USA; Miles und Iben (1999) für Großbritannien sowie Fehr (2000) und Hirte (2002) für Deutschland.

${ }^{8}$ Im Gegensatz zu den modellendogenen Kapital- und Güterströmen wird Migration durch das Bevölkerungsmodell exogen vorgegeben.
} 
Im Haushaltssektor maximieren die Haushalte unter vollkommener Voraussicht den aus ihrem Konsum erzielten Nutzen über das gesamte Leben. Im Mittelpunkt steht dabei die sogenannte Konsumglättung: Die Haushalte verteilen ihren Konsum möglichst gleichmäßig über den Lebenszyklus. Da im Alter kein Erwerbseinkommen mehr erzielt wird und die staatlich organisierte umlagefinanzierte Rente niedriger ausfällt als das letzte Erwerbseinkommen, bauen die Haushalte während ihres Erwerbslebens Ersparnisse auf, um einen deutlichen Konsumrückgang im Ruhestand zu vermeiden. Wegen der Bevölkerungsalterung wird die umlagefinanzierte gesetzliche Rente in Zukunft deutlich bescheidener werden, und so verstärkt sich die Notwendigkeit zur privaten Vorsorge weiter. Dieser Effekt wird in unserem Modell durch entsprechende Prognosen des Beitragssatzes und der Ersatzquote der gesetzlichen Rentenversicherung abgebildet (vgl. die Diskussion des Staatssektors weiter unten). ${ }^{9}$

Um der langfristigen demographischen Entwicklung der Bevölkerungsstruktur gerecht zu werden, unterscheiden wir zwei charakteristische Lebensabschnitte der Individuen: Erwerbsleben und Ruhestand. Der Anteil der zu einem bestimmten Zeitpunkt erwerbstätigen Bevölkerung und das Renteneintrittsalter werden in der Erwerbstätigenprognose geschlechts- und kohortenspezifisch festgelegt. Die Anzahl der sich im Erwerbsleben bzw. im Ruhestand befindenden Personen variiert gemäß der durch verschiedene Geburtenraten prognostizierten Bevölkerungsentwicklung. ${ }^{10}$ Unser Modell ist daher in der Lage, den Einfluss demographischer Veränderungen auf das Arbeitsangebot, die Güternachfrage und damit auf den Produktionssektor im Detail abzubilden.

Kinder erzielen noch kein eigenes Erwerbseinkommen und erhalten auch keine staatlichen Transfers, die in der Größenordnung mit der gesetzlichen Rente vergleichbar wären. Ihr Konsum und ihre Ausbildungen werden vielmehr von den Eltern finanziert. Kinder werden deshalb nicht als selbstständige Entscheidungsträger modelliert. Eltern berücksichtigen jedoch den Konsum ihrer Kinder bei der Konsum-und Sparentscheidung. Hierzu erweitern wir die Budgetrestriktion jedes Haushalts um den Konsum der Kinder, die diesem Haushalt anhand der altersspezifischen Fertilitätsraten der Frauen zugeordnet sind. Der Konsum eines jeden Kindes wird durch einen Aufschlagsfaktor auf den Konsum eines Erwachsenen berechnet. Berechnungen des Kinderkonsums ausgedrückt in Einheiten des Elternkonsums ergeben einen Skalierungsfaktor von 0.36. ${ }^{11}$ Demnach konsumiert ein Kind durchschnittlich 36 Prozent des Konsums eines Erwachsenen. Dieser Betrag entspricht ca. 309 Euro pro Monat.

Eine solche rein ökonomische Berücksichtigung der Kinder mag enttäuschen. Empirisch können wir jedoch nur die monetär quantifizierbaren relevanten Größen einigermaßen zuverlässig erfassen. Daher sehen wir von einer Abbildung des Nutzens von Kindern oder ihres Konsums ab. ${ }^{12}$

\footnotetext{
${ }^{9}$ Andere Sparmotive, z.B. eine geplante Vererbung und die Absicherung gegen Langlebigkeit oder unvorhersehbare Ereignisse, werden in unserem Modell nicht berücksichtigt.

${ }^{10}$ Weitere Einflussfaktoren sind die Erwerbsbeteiligung der Frauen und die gesamtwirtschaftliche Arbeitslosenquote, bezüglich derer wir den mittleren Projektionen von Birg und Börsch-Supan (1999) folgen.

${ }^{11}$ Diese Berechnungen basieren auf den Daten der Einkommens- und Verbrauchsstichprobe (EVS) 1993 zum Konsum von Kindern und Erwachsenen, die aufbereitet und nach verschiedenen Haushaltstypen differenziert in Hertel (1998) zu finden sind.

${ }^{12}$ Kotlikoff, Smetters und Walliser (2001) berücksichtigen in einem ansonsten sehr ähnlichen Ansatz den Konsum der Kinder im Nutzen der Eltern, wobei die Frage der Gewichtung jedoch umstritten bleibt (vgl. auch Brooks, 2002). Barro und Becker (1988) modellieren die „Qualität“ von Kindern, die an ihrem Humankapital gemessen wird - auch dies ist umstritten und in einem konkreten Simulationsmodell schwer umzusetzen.
} 
Der Produktionssektor besteht aus einem repräsentativen Unternehmen je Land. Bei gegebenem Einsatz der Produktionsfaktoren Arbeit und Kapital und einer gegebenen Technologie werden das BNE sowie der Lohn- und Zinssatz im Modell bestimmt. Wir nehmen zunächst an, dass die Produktivität der Faktoren exogen mit einer konstanten Rate wächst. Alternativ betrachten wir auch - in stilisierter Form - den Einfluss endogenen Wachstums. Dabei wird unterstellt, dass mit einem geringeren Alter der Erwerbstätigen auch das durchschnittliche Humankapital einer Gesellschaft steigt. Das Humankapital bestimmt wiederum die Produktivität, die dann endogen das gesamtwirtschaftliche Wachstum des Modells festlegt. ${ }^{13}$ Nach dieser Hypothese steigt also mit sinkendem Durchschnittsalter der Erwerbstätigen das Produktivitätswachstum der Volkswirtschaft. ${ }^{14}$

Wir beschränken uns auf diese stilisierte Abbildung endogenen Wachstums, obwohl der Aufbau von Humankapital in der Realität sehr vielschichtig ist. Alle Wirkungskanäle, die zu höherem Wachstum führen, können kaum in einem solchen Modell berücksichtigt werden. Zwar gibt es die theoretische Modellierung endogenen Wachstums durch einen „Learning-bydoing“-Ansatz, der auf Lucas (1988) zurückgeht und z.B. von Fougère und Mérette (1999) umgesetzt ist, oder den Ansatz des ,learning and doing“ nach Romer (1986). Jedoch ist es nicht möglich, diese theoretischen Modelle zuverlässig mit quantitativen Parametern zu kalibrieren.

Die Aufgabe des Staatssektors ist es, das Rentenversicherungssystem und alle weiteren Sozialversicherungsleistungen zu organisieren. Dazu gehört die Erhebung der Rentenversicherungs-, Arbeitslosen-, Kranken- und Pflegeversicherungsbeiträge sowie der Einkommensteuer, wobei die Einkommensteuer im Modell zur Finanzierung der staatlichen Zuschüsse zur Rentenversicherung und des allgemeinen Staatskonsums verwendet wird. Von einer Modellierung der übrigen Staatsausgaben sehen wir ab. Das Rentenniveau der umlagefinanzierten gesetzlichen Rentenversicherung (GRV) ist exogen und den Angaben des Bundesarbeitsministeriums entnommen. Der Beitragssatz ergibt sich dann aus der Budgetgleichung der GRV und den Bundeszuschüssen. Der Einstieg in eine teilweise kapitalgedeckte Altersvorsorge wird im folgenden nicht explizit modelliert, sondern ergibt sich aus dem oben beschriebenen Sparverhalten der Haushalte. ${ }^{15}$

In unserem idealtypischen Kapitalmarkt bestimmen sich Zins und internationale Kapitalströme dadurch, dass Kapital so lange in das Land mit höheren Zinsen fließt, bis die Zinsdifferenz wieder ausgeglichen ist. Da wir von Kapitalmarktunvollkommenheiten abstrahieren, sind sowohl der Zins als auch der Nettolohnsatz in allen betrachteten Ländern gleich. Der Zins bestimmt also nicht nur die Kapitalakkumulation im eigenen Land, sondern auch die Kapitalanlagen im Ausland und damit die Kapitalströme, die zwischen den betrachteten Ländern fließen. Diesen Zusammenhang nutzen wir bei der Lösung des Modells aus, indem die Berechnungen so lange iteriert werden, bis alle Märkte in allen Ländern geräumt sind.

\footnotetext{
${ }^{13}$ Die Frage, ob eine größere und/oder jüngere Bevölkerung positive oder negative Auswirkungen auf die Produktivität einer Volkswirtschaft hat, ist umstritten. Siehe z.B. Becker, Edward und Murphy (1999).

${ }^{14}$ Statt das Humankapital abhängig vom Durchschnittsalter der Erwerbstätigen zu machen, kann auch die Anzahl der Kinder als Maß für das zukünftige Humankapital einer Gesellschaft verwendet werden. Die Ergebnisse unterscheiden sich jedoch qualitativ kaum.

${ }^{15}$ Zur Einführung der kapitalgedeckten Altersversorgung siehe auch Börsch-Supan, Heiss, Ludwig und Winter (2002).
} 


\subsection{Kritik}

Jede modellhafte Abbildung der Realität ist eine starke Vereinfachung. Während die zukünftige demographische Entwicklung sehr detailliert abgebildet und die für Deutschland so wichtige Verflechtung nationaler Kapitalmärkte berücksichtigt wird, blenden wir eine Reihe von Aspekten aus, die für diese Studie nicht zentral sind: ${ }^{16}$

(1) Größen wie das Arbeitsangebot oder auch die Geburtenentscheidung sind exogen durch die Bevölkerungs- und Erwerbstätigenentwicklung vorgegeben. Daher bleiben mögliche Rückkopplungseffekte zwischen der Geburtenentscheidung einer Frau oder eines Paares und dem Arbeitsangebot einer Frau oder Effekte ausgehend von den Sozialversicherungssystemen auf diese Größen unberücksichtigt. ${ }^{17}$

(2) In unserem Modell wird von Marktfriktionen auf dem inländischen Arbeitsmarkt und jeglichen Kapitalmarktunvollkommenheiten wie z. B. Kreditrestriktionen abgesehen. Die Annahme vollkommener Kapitalmärkte ist bei der Abbildung von Kapitalströmen innerhalb der EU jedoch nicht sehr restriktiv.

(3) Es wird unterstellt, dass Haushalte vorausschauend agieren und keinerlei Unsicherheiten bezüglich der Zukunft existieren. Dazu gehört zum einen auf individueller Ebene das Risiko der Langlebigkeit, gegen das sich Individuen durch Vorsichtssparen absichern würden. Zum anderen bleibt auf aggregierter Ebene das Risiko auf den Finanzmärkten unbeachtet.

Diese Vereinfachungen haben aus unserer Sicht keine wesentlichen Auswirkungen auf die zentralen Ergebnisse dieser Studie, da sie nur indirekt über Effekte zweiter Ordnung auf den uns eigentlich interessierenden Zusammenhang zwischen Geburtenrate und Wirtschaftswachstum einwirken. ${ }^{18}$

\section{Welche Auswirkungen hat die Bevölkerungsentwicklung auf die deutsche Volkswirt- schaft?}

Wie würde sich die deutsche Volkswirtschaft entwickeln, wenn die Geburtenrate auf 1,8 Kinder je Frau ansteigt? Oder: Was wären die Folgen einer noch weiter sinkenden Geburtenrate? Zur Beantwortung dieser Fragen analysieren wir insbesondere die Auswirkung der Bevölkerungsentwicklung auf das Niveau des BNE pro Kopf und sein Wachstum. Dabei gehen wir zunächst davon aus, dass der technologische Fortschritt unabhängig von der Bevölkerungsstruktur ist. Im Anschluss vergleichen wir dann die Ergebnisse dieses Ausgangsmodells mit einem Szenario, in dem - wie in Abschnitt 3.2 beschrieben - die Produktivität an die Bevölkerungsstruktur gekoppelt ist.

\subsection{Exogenes Produktivitätswachstum}

Wie aus der in Abschnitt 2 dargestellten Bevölkerungs- und Erwerbstätigenprognose hervorgeht, sind in den ersten 20 Jahren nach einem Anstieg der Geburtenrate mehr Kinder vorhanden die noch nicht erwerbstätig sind, sondern von der Gesellschaft versorgt und ausgebildet

\footnotetext{
${ }^{16}$ Siehe dazu auch die Übersicht bei Kotlikoff (1998).

${ }^{17}$ Sinn (1998), Barro und Becker (1988) sowie Cigno (1991) weisen auf negative Effekte hin, die Sozialversicherungssysteme auf die Geburtenentscheidung und Humankapitalbildung haben können. Zum Zusammenhang zwischen technologischem Fortschritt und Bevölkerungswachstum siehe auch Fernandez-Villaverde (2001). Eine Übersicht über die theoretische Literatur zur Geburtenentscheidung gibt Tamura (2000).

${ }^{18}$ Wir arbeiten gegenwärtig daran, die Größenordnung dieser Effekte abzuschätzen.
} 
werden müssen. Diese Entwicklung schlägt sich im Wirtschaftswachstum nieder. Es werden mehr Ressourcen für die Kinder benötigt, wodurch die Kapitalakkumulation teilweise gedämpft wird, was sich wiederum auf die Produktion auswirkt. Folglich ergeben sich bei einer Erhöhung der Geburtenrate zunächst Wachstumseinbußen. Abgesehen davon nehmen die ProKopf-Größen schon allein rein rechnerisch ab, da das erwirtschaftete BNE auf eine größere Anzahl von Personen - nämlich auch auf die zusätzlichen Kinder - aufgeteilt werden muss.

\section{Abbildung 4: Direkter und indirekter Beitragssatz zur Rentenversicherung}

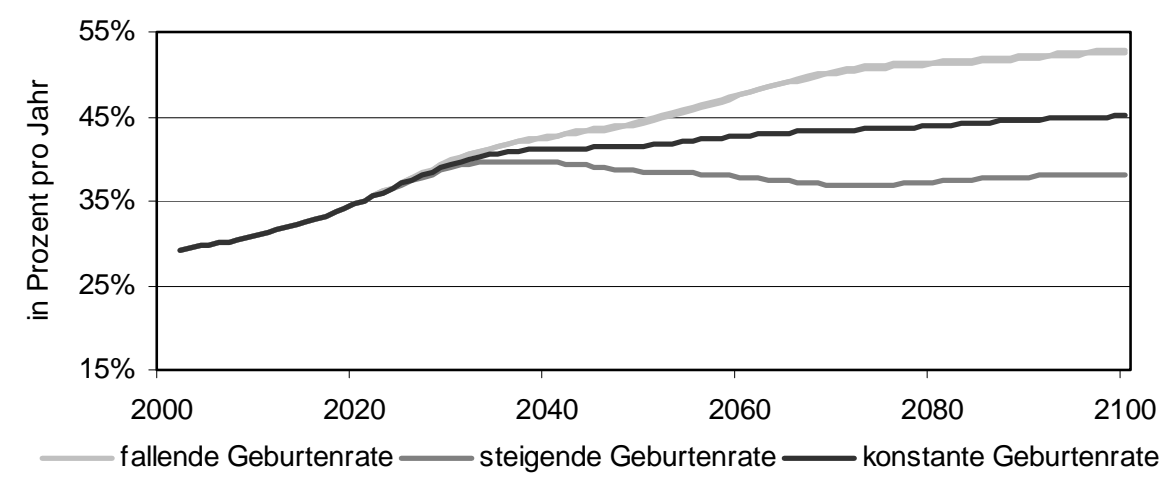

Abbildung 5: Wachstumsrate des BNE pro Kopf

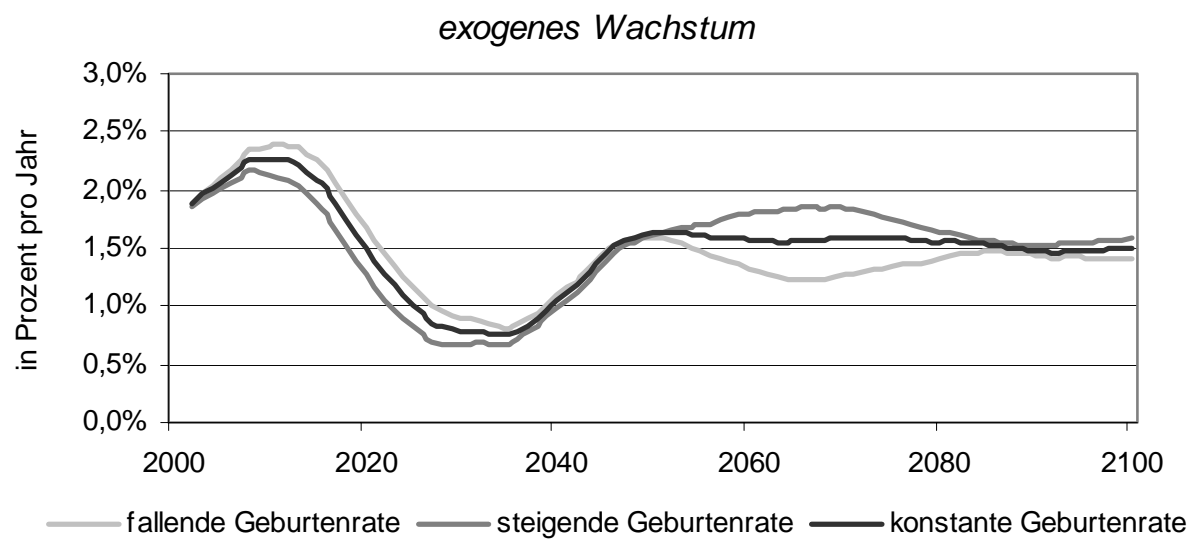

Diese anfänglichen Wachstumseinbußen sind erst überwunden, wenn die Kinder ausgebildet sind und mit 20 Jahren ins Erwerbsleben eintreten, also frühestens ab 2035. Doch gerade dann erreichen das Alterungsproblem und die Krise der gesetzlichen Kranken- und Rentenversicherung ihren Höhepunkt. ${ }^{19}$ Selbst ein durchaus dramatischer Anstieg der Geburtenrate auf 1,8 Kinder je Frau kann also zu keiner Milderung des kurzfristigen Alterungsproblems führen.

Nach diesem Übergangszeitraum sind die positiven Auswirkungen einer steigenden Geburtenrate spürbar. Aufgrund der höheren Anzahl von Kindern, die in das Erwerbsleben eintreten, wächst die Anzahl der Erwerbstätigen schneller als die übrige Bevölkerung. Die Alterslast kann somit auf mehr Schultern verteilt werden. Ablesbar ist dies sehr deutlich an der Entwick-

\footnotetext{
${ }^{19}$ Die Konsequenzen des Alterungsprozesses für das soziale Sicherungssystem in Deutschland werden viel diskutiert. Siehe Birg (2001), S. 170-194 und Börsch-Supan (2002).
} 
lung des Gesamtbeitragssatzes ${ }^{20}$ zur Rentenversicherung in Abbildung 4: Bis zur Zuspitzung des Altersproblems in den Jahren 2035 bis 2040 ist der Beitragssatz für alle Szenarien gleich. Bereits im Jahr 2060 ist der Beitragssatz bei steigender Geburtenrate immerhin 5 Prozentpunkte niedriger als im Ausgangsszenario mit konstanter Geburtenrate. Neben dem höheren Erwerbsangebot führt unter anderem diese Entlastung dazu, dass die Wachstumsrate des BNE pro Kopf bei steigender Geburtenrate zwischen 2035 und 2080 zeitweise deutlich höher ist als im Vergleichsszenario, in dem die Geburtenrate konstant bleibt (siehe Abbildung 5).

Langfristig jedoch geht dieser positive Effekt wieder zurück. Wie wir gesehen haben, führt die höhere Anzahl der Erwerbstätigen in den Jahren 2035 bis 2060 zu einem höheren Wachstum, da die Anzahl der Erwerbstätigen relativ zur Bevölkerung schneller wächst. Hinzu kommt, dass die ältere Bevölkerung der Babyboomjahre gerade in dieser Zeit verstirbt. Nach 2075 etwa erreicht die erste Welle der aus einer höheren Geburtenrate resultierenden Erwerbstätigenbevölkerung das Rentenalter. Bei steigender Geburtenrate schrumpft folglich nun auch die Anzahl der Rentner weniger stark (Abbildung 2) und der Rentnerquotient steigt wieder leicht an (Abbildung 3), bis er etwa im Jahr 2100 ein konstantes Niveau erreicht. Unter diesen Bedingungen kommt auch der gegenläufige Effekt, nach dem zusätzliche Arbeitnehmer nur unterproportional zur Produktion beitragen, wieder stärker zum Tragen. ${ }^{21}$ Zum Ende unseres Jahrhunderts gleichen sich daher bei konstantem technologischen Fortschritt die Wachstumsraten pro Kopf in etwa wieder an. Der positive Pro-Kopf-Wachstumseffekt einer höheren Geburtenrate verpufft also langfristig.

\section{Abbildung 6: BNE pro Kopf}

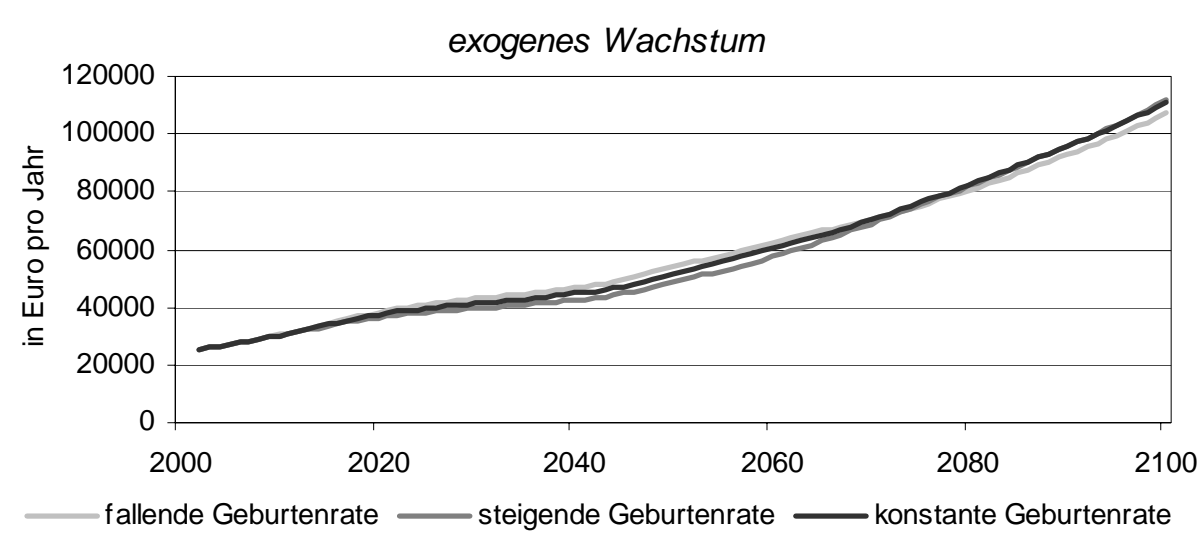

Ähnlich sind die in Abbildung 6 gezeigten Auswirkungen einer höheren Geburtenrate auf das Niveau des BNE pro Kopf. Schon rein optisch sind Unterschiede in den Verläufen schwer $\mathrm{zu}$ erkennen. Bei genauerer Betrachtung sind jedoch dieselben qualitativen Auswirkungen erkennbar, die wir auch beim Wirtschaftswachstum beobachtet haben. Im Fall einer steigenden Geburtenrate ist das BNE pro Kopf zunächst niedriger als im Vergleichsszenario. Allerdings hält der anfänglich negative Effekt länger an, da zunächst die Wachstumseinbußen im Niveau des BNE aufgeholt werden müssen. Erst in den 80er Jahren dieses Jahrhunderts ist ein schwacher positiver Effekt auf das Niveau des BNE pro Kopf absehbar.

\footnotetext{
${ }^{20}$ Der Gesamtbeitragssatz ist die Summe aus direktem Beitragssatz und den indirekten, aus dem allgemeinen Steueraufkommen finanzierten Zuschüssen zur gesetzlichen Rentenversicherung; vgl. beispielsweise BörschSupan, Heiss, Ludwig und Winter (2002).

${ }^{21}$ Dieses Argument geht auf Solow (1956) zurück. Vgl. oben Abschnitt 1 sowie ausführlich Cutler et al. (1990).
} 


\subsection{Endogenes Produktivitätswachstum durch Akkumulation von Humankapital}

Die unter der Annahme exogenen Wachstums erzielten ernüchternden Ergebnisse des vorangehenden Abschnitts gelten jedoch nicht, wenn die Möglichkeit berücksichtigt wird, dass die künftig geborenen Kinder besser ausgebildet werden, so dass sie den technischen Fortschritt beschleunigen. Um diesen Effekt zu illustrieren, verwenden wir in diesem Abschnitt eine Variante unseres Simulationsmodells, die endogenes Wachstum zulässt. Wie in Abschnitt 3.1 beschrieben, nehmen wir sehr stilisiert an, dass die Produktivität der Arbeitnehmerschaft höher ist, wenn ihr Durchschnittsalter niedriger ist. Diese Hypothese entspricht einer geläufigen Einschätzung. Ob sie tatsächlich empirisch belegbar ist, ist allerdings unklar. Die folgenden Simulationen sollten also eher als Parabel für die Effekte einer besseren Ausbildung verstanden werden.

Die Abbildungen 7 und 8 zeigen unsere Ergebnisse. Wenn zusätzlich zu einer steigenden Geburtenrate auch die Produktivität der Arbeitnehmerschaft dank eines höheren Humankapitals wächst, ergeben sich zwei wesentliche Veränderungen zu den Ergebnissen bei exogenem Wachstum. Erstens liegt das Wirtschaftswachstum pro Kopf bei endogenem Wachstum und steigender Geburtenrate deutlich über dem Wirtschaftswachstum bei exogenem Wachstum (siehe Abbildung 7). Zweitens gilt dieser positive Effekt nun auch über das Jahr 2085 hinaus, da eine höhere Geburtenrate langfristig zu einer jüngeren und - gemäß unserer Annahme produktiveren Erwerbsbevölkerung führt. In diesem Fall trägt ein zusätzlicher jüngerer Arbeitnehmer im Vergleich zu einem älteren Arbeitnehmer überproportional zur Produktion bei, da der nun besser ausgebildete zusätzliche Arbeitnehmer die abnehmenden Grenzerträge mehr als kompensieren kann. Im Fall mit exogenem Wachstum werden dagegen junge und alte Arbeitnehmer gleich bewertet, und ein zusätzlicher Arbeitnehmer trägt wegen der abnehmenden Grenzproduktivität nur unterproportional zur Produktion bei (vgl. Abschnitt 4.1).

\section{Abbildung 7: Wachstumsrate des BNE pro Kopf}

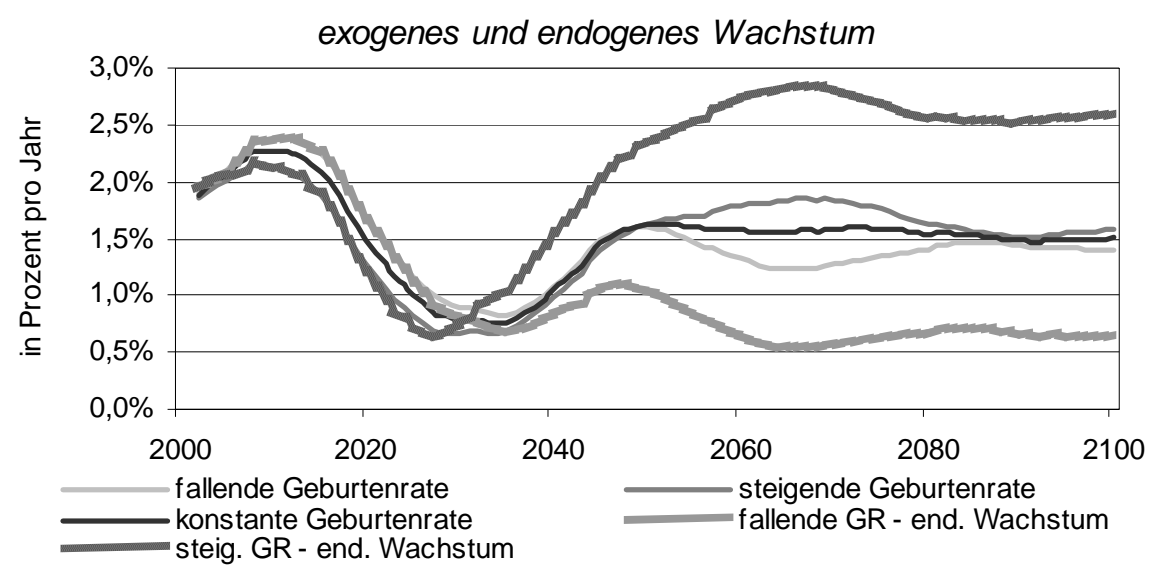

Auch bezüglich des BNE pro Kopf ist bei endogenem Wachstum ein qualitativ und quantitativ deutlich positiveres Ergebnis sichtbar (Abbildung 8): Wenn das aus einer höheren Geburtenrate resultierende niedrigere Erwerbstätigenalter $\mathrm{zu}$ einem positiven Humankapitaleffekt führt, liegt das BNE pro Kopf ab ungefähr 2055 deutlich über dem Niveau bei exogenem Wachstum und steigender Geburtenrate. Entscheidend ist auch hier wiederum, dass dieser Effekt über das Jahr 2085 hinaus reicht und daher langfristig gilt. Darüber hinaus ist der positive Effekt nicht nur größer und permanent, sondern tritt auch ungefähr fünf Jahre früher ein. 


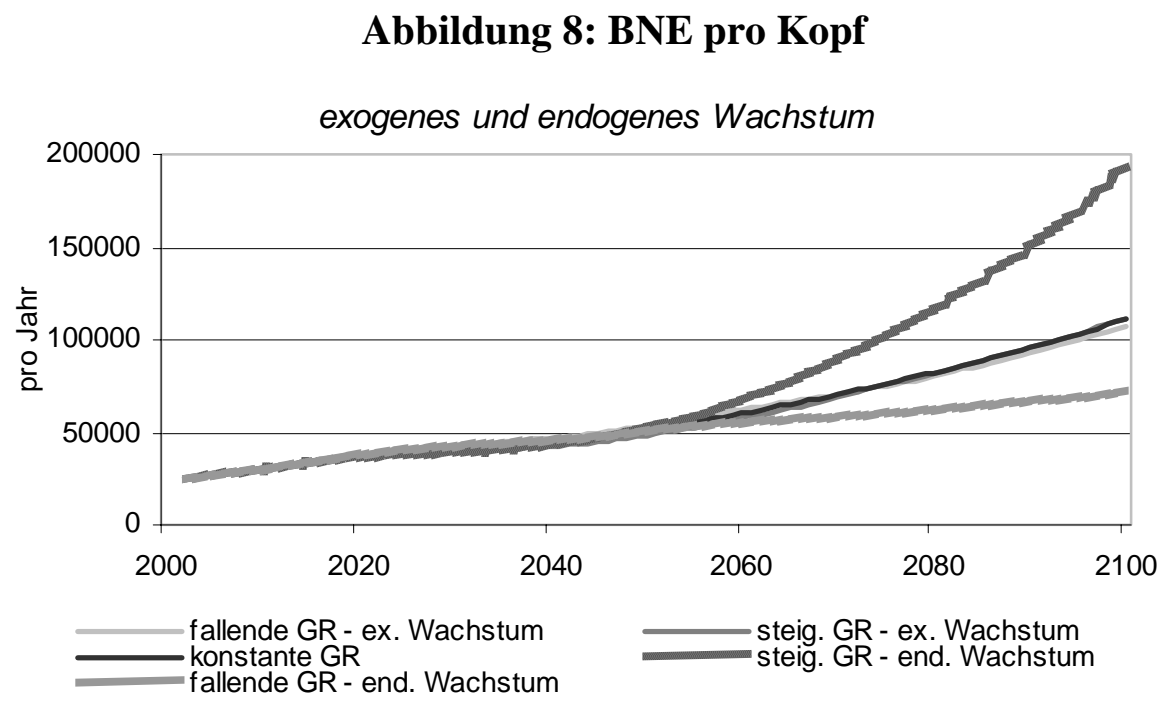

Nach den zunächst sehr ernüchternden Ergebnissen einer höheren Geburtenrate bei exogenem Wachstum, machen diese Ergebnisse deutlich, dass der Ausbildung der Kinder und Jugendlichen eine Schlüsselrolle bei der Bewältigung des demographischen Wandels zukommt.

\section{Schlussfolgerungen}

Hilft eine höhere Geburtenrate, die Folgen der Alterung zu dämpfen? Die Ergebnisse der Wirtschaftstheorie sind keinesfalls eindeutig, auch wenn die Idee - „Wenn wir zu viele Alte haben, brauchen wir mehr Kinder, um dies wieder auszugleichen“ - plausibel erscheint. Auch die vorliegende quantitative Studie kommt zu einem differenzierten Ergebnis: Eine langfristige Stärkung des Bruttonationaleinkommens pro Kopf entsteht durch eine höhere Geburtenrate nur dann, wenn die zusätzlich geborenen Kinder auch besser ausgebildet werden. Entscheidend für ein langfristiges Wachstum ist daher das zukünftige Humankapital.

Daher ziehen wir aus unserer Untersuchung drei wichtige wirtschaftspolitische Schlussfolgerungen:

(1) Die Ausbildung unserer Kinder ist die Quintessenz künftigen Wirtschaftswachstums. Das alternde Deutschland braucht besser ausgebildete und daher hoch produktive Kinder. Der Motor des zukünftigen Wachstums ist die Ausbildung und Erziehung in Familie, Schule, Universität und beruflicher Weiterbildung.

(2) Da die Anzahl der neugeborenen Kinder das Pro-Kopf-BNE kaum beeinflusst, lassen sich ökonomische Gründe für eine Förderung einer höheren Geburtenrate nicht ohne weiteres ableiten. Die vorliegende Untersuchung abstrahiert allerdings von den Problemen des heutigen Familienleistungsausgleichs. Bei diesem ist zwar unklar, ob er hoch genug angesetzt ist, doch mehr als den Ausgleich der Lasten und Leistungen können Ökonomen nicht fordern.

(3) Die Dauer der Übergangszeit, nach deren Ablauf sich eine höhere Geburtenrate in mehr und besser ausgebildeten Erwerbstätigen niederschlägt, die in die Renten- und anderen Sozialversicherungskassen einzahlen können, ist sehr lang. Von daher haben weitere Reformen unserer Sozialversicherungen nach wie vor höchste Priorität, da nur sie die sozialpolitischen Probleme einer alternden Babyboomgeneration kurz- und mittelfristig lösen können. 


\section{Literaturverzeichnis}

Altig, D., A.J. Auerbach, L.J. Kotlikoff, K.A. Smetters und J. Walliser (2001): Simulating fundamental tax reform in the United States. American Economic Review, 91, 574595.

Auerbach, A.J. und L.J. Kotlikoff (1987): Dynamic Fiscal Policy. Cambridge, MA: Cambridge University Press.

Becker, G. S. und R. J. Barro (1988): A reformulation of the economic theory of fertility. Quarterly Journal of Economics, 103, 1-25.

Becker, G., L. Edward und K. M. Murphy (1999): Population and economic growth. American Economic Review, Papers \& Proceedings, 89:2, 145-152.

Birg, H. (2001): Die demographische Zeitenwende - Der Bevölkerungsrückgang in Deutschland und Europa. München: C. H. Beck.

Birg, H. und A. Börsch-Supan (1999): Für eine neue Aufgabenteilung zwischen gesetzlicher und privater Altersversorgung. Berlin: Gesamtverband der Versicherungswirtschaft.

Bloom, D. E., Canning, D. und Sevilla, J. (2001): Demographic change and economic growth in Asia. Working Paper No. 15, Center for International Development, Harvard University, Cambridge, MA.

Börsch-Supan, A. (1995): Die Rolle von Direktinvestitionen bei der regionalen Entwicklung eines alternden Europas. In: B. Gahlen, H. Hesse und H.-J. Ramser (Hg.), Standort und Region: Neue Ansätze zur Regionalökonomik, Tübingen: J.C.B. Mohr (Paul Siebeck).

Börsch-Supan, A. (2002): Nach der Reform ist vor der Reform: Weitere Schritte für eine nachhaltige Reform der Altersvorsorge in Deutschland. MEA Discussion Paper No. 15-2002, Mannheim Research Institute for the Economics of Aging (MEA), Universität Mannheim.

Börsch-Supan, A., B. Berkel, A. Ludwig und J. Winter (2002): Geburten, Wachstum und Alterung - Gutachten für das Heidelberger Büro für Familienfragen und Soziale Sicherheit. Mannheim Research Institute for the Economics of Aging, Universität Mannheim.

Börsch-Supan, A., A. Ludwig und J. Winter (2002): Aging and international capital flows. In: A. Auerbach und H. Hermann (Hg.): Aging, Financial Markets and Monetary Policy, Heidelberg: Springer, 55-83.

Börsch-Supan, A., F. Heiss, A. Ludwig und J. Winter (2002): Pension reform, capital markets, and the rate of return. Erscheint in: German Economic Review.

Brooks, R. (2002): Population Aging and global capital flows in a parallel universe, Working Paper No. 00/151, International Monetary Fund, Washington, D.C.

Cutler, D. M., J. M. Poterba, L.M. Sheiner und L.H. Summers (1990): An aging society: Opportunity or challenge? Brookings Papers on Economic Activity, No. 1, 1-73.

De Nardi, M., S. Imrohoroglu und T.J. Sargent (1999): Projected U.S. demographics and social security. Review of Economic Dynamics, 2, 575-615.

Diamond, P. A. (1965): National debt in a neoclassical growth model. American Economic Review, 55, 1126-1150. 
Fehr, H. (2000): Pension reform during the demographic transition. Scandinavian Journal of Economics, 102, 419-443.

Fernandez-Villaverde, J. (2001): Was Malthus right? Economic growth and population dynamics, Unveröffentlichtes Manuskript, University of Pennsylvania.

Fougère, M. und M. Mérette (1999): Population aging and economic growth in seven OECD countries. Economic Modelling, 16, 411-427.

Guest, R. S. und I. M. McDonald (2002): Would a decrease in fertility be a threat to living standards in Australia? Australian Economic Review, 35, 29-44.

Hertel, J. (1998): Aufwendungen für den Lebensunterhalt von Kindern. Wirtschaft und Statistik, Nr. 6, 523-533.

Hirte, G. (2002): Welfare and macroeconomic effects of the German pension acts of 1992 and 1999: A dynamic CGE study. German Economic Review, 3, 81-106.

Hurd, M. (1989): Mortality risk and bequest. Econometrica, 57, 173-209.

Kottlikoff, L. J. (1998): The A-K model - Its past, present and future. Working Paper No. 6684, National Bureau of Economic Research (NBER), Cambridge, MA.

Kotlikoff, L. J., K. Smetters und J. Walliser (1999): Privatizing social security in the United States: Comparing the options. Review of Economic Dynamics, 2, 532-574.

Kotlikoff, L. J., K. Smetters und J. Walliser (2001): Finding a way out of America's demographic dilemma. Working Paper No. 8258, National Bureau of Economic Research (NBER), Cambridge, MA.

Lucas, R. E. (1988): On the mechanics of economic development. Journal of Monetary Economics, 22, 3-42.

Ludwig, A. (2002): Rentenreformen im globalen Kontext. Frankfurt: Peter Lang.

Miles, D. und A. Iben (2000): The reform of pension systems: Winners and losers across generations in the United Kingdom and Germany. Economica, 67, 203-228.

OECD (1999): Labor Force Statistics. Paris: Organization for Economic Co-Operation and Development (OECD).

Romer, P. (1986): Increasing returns and long-run growth. Journal of Political Economy, 94, 1002-1037.

Samuelson, P. A. (1958): An exact consumption-loan model of interest with or without the social contrivance of money. Journal of Political Economy, 66, 467-482.

Sinn, H. W. (1998): The pay-as-you-go pension system as a fertility insurance and enforcement device. Working Paper No. 6610, National Bureau of Economic Research (NBER), Cambridge, MA.

Solow, R. (1956): A contribution to the theory of economic growth. Quarterly Journal of Economics, 70, 65-94.

Statistisches Bundesamt (2001): Statistisches Jahrbuch 2001 für die Bundesrepublik Deutschland. Wiesbaden: Statistisches Bundesamt.

Statistisches Bundesamt (1993): Sonderreihe mit Beiträgen für das Gebiet der ehemaligen DDR, Heft 3: Bevölkerungsstatistische Übersichten 1946 bis 1989. Wiesbaden: Statistisches Bundesamt. 
Tamura, R. (2000): Growth, fertility and human capital: A survey. Spanish Economic Review, 2, 183-229.

UN (2000): World Population Prospects: The 1998 Revision. New York: United Nations, Department of Economic and Social Affairs, Population Division.

Weil, D. N. (1999): Population growth, dependency, and consumption. American Economic Review, Papers \& Proceedings, 89:2, 251-254. 


\section{Discussion Paper Series}

Mannheim Research Institute for the Economics of Aging Universität Mannheim

To order copies, please direct your request to the author of the title in question.

\begin{tabular}{|c|c|c|c|}
\hline Nr. & Autoren & Titel & Jahr \\
\hline $11-02$ & Axel Börsch-Supan & Labor market effects of population aging & 02 \\
\hline $12-02$ & Axel Börsch-Supan & $\begin{array}{l}\text { Kann die Finanz- und Sozialpolitik die } \\
\text { Auswirkungen der Bevölkerungsalterung auf den } \\
\text { Arbeitsmarkt lindern? }\end{array}$ & 02 \\
\hline $13-02$ & $\begin{array}{l}\text { Florian Heiß, } \\
\text { Jens Köke }\end{array}$ & $\begin{array}{l}\text { Dynamics in ownership and firm survival: } \\
\text { Evidence from corporate Germany }\end{array}$ & 02 \\
\hline $14-02$ & Axel Börsch-Supan & $\begin{array}{l}\text { "Global Aging“ an der Jahrtausendwende: } \\
\text { Die demographischen Herausforderungen des } \\
\text { 21. Jahrhunderts }\end{array}$ & 02 \\
\hline $15-02$ & Axel Börsch-Supan & $\begin{array}{l}\text { Nach der Reform ist vor der Reform: Weitere } \\
\text { Schritte für eine nachhaltige Reform der } \\
\text { Altersvorsorge in Deutschland }\end{array}$ & 02 \\
\hline $16-02$ & Florian Heiss & Specification(s) of Nested Logit Models & 02 \\
\hline 17-02 & Axel Börsch-Supan & $\begin{array}{l}\text { What We Know and What We Do NOT Know } \\
\text { About the Willingness to Provide Self-Financed } \\
\text { Old-Age Insurance }\end{array}$ & 02 \\
\hline $18-02$ & $\begin{array}{l}\text { Axel Börsch-Supan, } \\
\text { Agar Brugiavini }\end{array}$ & Savings: The Policy Debate in Europe & 02 \\
\hline 19-02 & $\begin{array}{l}\text { Axel Börsch-Supan, } \\
\text { Lothar Essig }\end{array}$ & Stockholding in Germany & 02 \\
\hline $20-02$ & $\begin{array}{l}\text { Axel Börsch-Supan, } \\
\text { Simone Kohnz, } \\
\text { Reinhold Schnabel }\end{array}$ & $\begin{array}{l}\text { Micro Modeling of Retirement Decisions in } \\
\text { Germany }\end{array}$ & 02 \\
\hline 21-02 & Joachim Winter & $\begin{array}{l}\text { The impact of pension reforms and demography } \\
\text { on stock markets }\end{array}$ & 02 \\
\hline $22-02$ & Axel Börsch-Supan & $\begin{array}{l}\text { Mehr Zuwanderung? } \\
\text { Zur Rolle des Auslands bei der Stabilisierung der } \\
\text { gesetzlichen Rentenversicherung in Deutschland }\end{array}$ & 02 \\
\hline $23-02$ & $\begin{array}{l}\text { Axel Börsch-Supan, } \\
\text { Florian Heiss, } \\
\text { Alexander Ludwig, } \\
\text { Joachim Winter }\end{array}$ & $\begin{array}{l}\text { Pension reform, capital markets, and the rate of } \\
\text { return }\end{array}$ & 02 \\
\hline 24-02 & $\begin{array}{l}\text { Axel Börsch-Supan, } \\
\text { Annamaria Lusardi }\end{array}$ & Saving Viewed from a Cross-National Perspective & 02 \\
\hline $25-02$ & $\begin{array}{l}\text { Barbara Berkel, } \\
\text { Axel Börsch-Supan, } \\
\text { Alexander Ludwig, } \\
\text { Joachim Winter }\end{array}$ & $\begin{array}{l}\text { Sind die Probleme der Bevölkerungsalterung } \\
\text { durch eine höhere Geburtenrate lösbar? }\end{array}$ & 02 \\
\hline & & & \\
\hline
\end{tabular}


